\title{
Interferometry Method as a Way to Find Nonlinear Optical Responses of Nanoparticles
}

\author{
S. Salmani ${ }^{a, b, *}$ N. Ghazyani ${ }^{a, b}$, A. Kavianfar ${ }^{a}$, M. Behravan ${ }^{a}$ And M.H. Majles Ara $^{a, b}$ \\ ${ }^{a}$ Photonics Lab, Department of Physics, Kharazmi University, Tehran, Iran \\ ${ }^{b}$ Research Institute of Applied Sciences, Kharazmi University, Karaj, Iran
}

(Received January 30, 2018; revised version October 1, 2018; in final form November 25, 2018)

\begin{abstract}
In this paper, for the first time, we introduce a simple way for finding the nonlinear optical properties of nanoparticles. The Michelson interferometer was used for calculating nonlinear refractive index. In comparison with conventional methods, the interferometry has high sensitivity and uses a single coherent beam that reduces the complexity and cost of experimental setup. The He-Ne laser with $75 \mathrm{~mW}$ laser irradiation at $632.8 \mathrm{~nm}$ was applied to different concentrations of $\mathrm{TiO}_{2}$ nanoparticles as a notable sample. By analysing the changes at interferometric patterns of different samples, the magnitude of $\left(n_{2}\right)$ was obtained in $10^{-8} \mathrm{~cm}^{2} / \mathrm{W}$ order. To confirm the interferometry results, the common Z-scan method was used to measure the nonlinear refractive index which reveals that the results are in good agreement with the interferometry method.
\end{abstract}

DOI: 10.12693/APhysPolA.136.62

PACS/topics: nonlinear refractive index, Michelson interferometer, Z-scan technique, nanoparticles

\section{Introduction}

In last few decades, the optical properties of nanoparticles have received considerable attention around the world [1-3]. There are different methods for evaluation of optical properties of nanoparticles. In this field of study, the interferometry method has not been applied yet. Because of the accuracy and high sensitivity, this method has attracted the attentions [4]. In this paper we used the Michelson interferometry to find the nonlinear refractive index for the first time. This method has been depicted in many literatures and was invented by Michelson [5]. A Michelson interferometer is a tool to produce interference between two beams of light. In addition, the Michelson interferometers are relatively simple in terms of operation, and possess the largest field of view for a specified wavelength and other applications [68]. To confirm the obtained results from interferometry method, the close Z-scan technique was applied to measure the nonlinear refractive index, too. This method is a very common tool for measurements of nonlinear index, both in sign and magnitude [9]. In this work, we study the nonlinear optical responses of $\mathrm{TiO}_{2}$ nanoparticles by the Michelson interferometry for the first time. $\mathrm{TiO}_{2}$ nanoparticles are known for their numerous and diverse applications in cases such as optoelectronic, photo catalysis, gas sensors, photo electrolysis, and photovoltaic cases $[10,11]$. Hence, finding the optical responses can lead to new applications or improve the current applications. To extend our results, the effect of concentration of aqueous $\mathrm{TiO}_{2}$ nanoparticles was investigated with Zscan and the Michelson interferometry methods, which will be described in detail in following sections.

*corresponding author; e-mail: salmani@khu.ac.ir

\section{Experimental}

\subsection{Materials}

We have synthesized anatase $\mathrm{TiO}_{2}$ nanoparticles by a sol-gel and hydrothermal process via modified barbe's method [12]. All materials were purchased from Merck and water was purified by distillation and filtration (Milli-Q). A quantity of titanium iso-propoxide and acetic acid were added into deionized (DI) water under vigorously stirring at room temperature. A white precipitate formed immediately. The stirring for $1 \mathrm{~h}$ was required to achieve a complete hydrolysis reaction. After adding a quantity of nitric acid, to achieve peptization, the mixture was heated to $80^{\circ} \mathrm{C}$ and stirred vigorously for $2 \mathrm{~h}$. The growth and crystallinity of these particles occurred under hydrothermal conditions in a titanium autoclave that was heated for $12 \mathrm{~h}$ in the $250^{\circ} \mathrm{C}$ temperature. Following this step, nitric acid was added and the dispersion was treated with a titanium ultrasonic horn (400 W, 20 times with $2 \mathrm{~s}$ pulses). Finally, we prepared various weight percentages of $\mathrm{TiO}_{2}$ nanoparticles at DI water. Figure 1 shows the UV-visible absorption spectra of $\mathrm{TiO}_{2}(5 \mathrm{wt} \%)$ that was measured using Perkin Elmer spectrophotometer. As it is clear from UV-vis spectrum, there is a bit absorption at the laser incident wavelength. Therefore, optical responses come mostly from refraction changes rather than absorption index.

\subsection{Experimental setup}

The experimental optical investigations need to have a nearly complete knowledge about different optical setups. For example, in the last few years, the optical properties of different samples were studied using the Raman spectroscopy [13-14]. Beside all these optical setups, the interferometry setup attracted more attentions. Many studies reported works on interferometry setups to measure the optical parameters of different types of materials, 


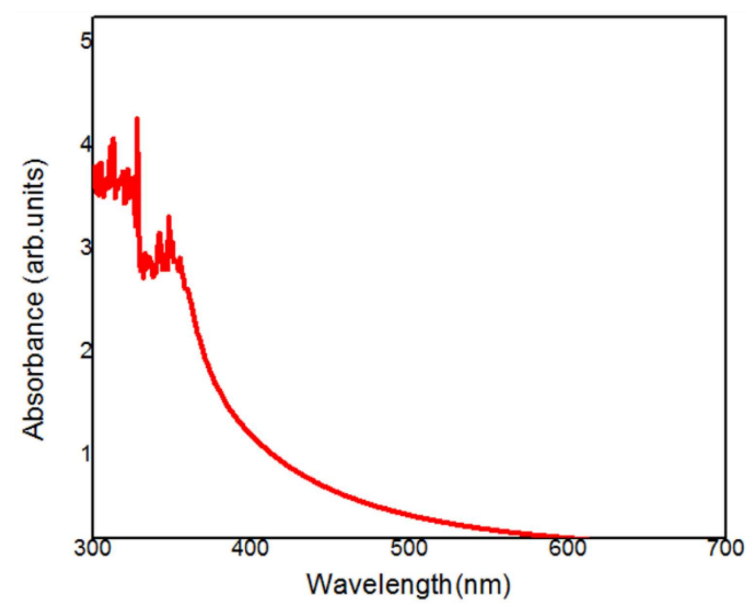

Fig. 1. UV-visible spectrum of $\mathrm{TiO}_{2}$ nanoparticles.

and they were in good agreement with other optical methods like Z-scan method [15, 16]. Because of the high precision results that have been obtained from interferometers and the novelty of this method, we have chosen the Michelson interferometer to study optical properties of aqueous $\mathrm{TiO}_{2}$ nanoparticles.

\subsubsection{Michelson interferometer}

Alongside the methods and setups to study the optical properties, we have used the Michelson interferometer to study aqueous $\mathrm{TiO}_{2}$ nanoparticles with four different concentrations. The advantages of interferometry method are high accuracy, wide spectral capability, and instrumentation simplicity. So, by using a simple but very high precision set up we may be able to study optical parameters such as nonlinear refractive index $\left(n_{2}\right)$.

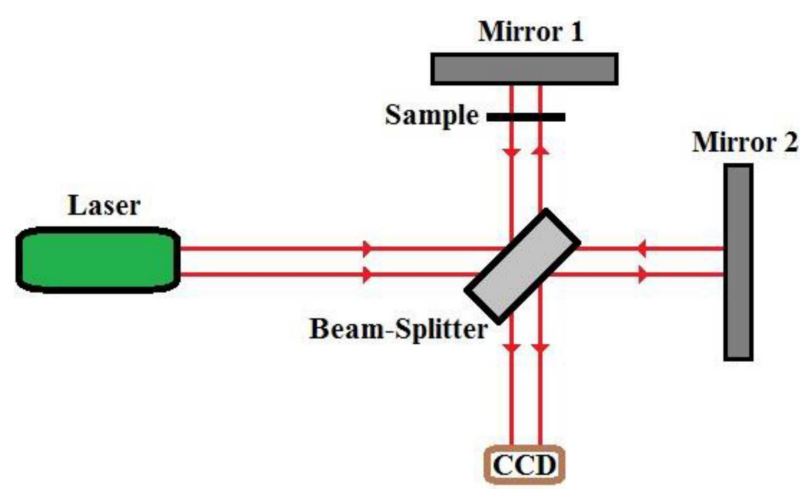

Fig. 2. The experimental setup of the Michelson interferometry.

Figure 2 shows the interferometry setup of the Michelson interferometer in detail. As we explained, this setup has simple but accurate components which could be applied to study laser-material interactions. In the Michelson interferometer, a beamsplitter and two mirrors are inserted into an orthogonal shape. A Gaussian coherent laser beam diverges via a lens and separates out into two light beams. As a matter of fact the Michelson interferometer is an amplitude-divided interferometer. So these two light beams travel along two paths and after reflection from the mirrors meet each other again in the beamsplitter which produces interference pattern. In fact, the main reason is that these two light beams experience two different paths because of inserting a medium with refractive index not equal to refractive index of air. Here, in this paper, we have used aqueous $\mathrm{TiO}_{2}$ nanoparticles as a medium to produce the fringe patterns.

The intensity of light in the fringes depends on the intensity of light in the first path and the intensity of light in the second path and a term that is function of phase shift between two light beams. So, the resultant intensity will be in the form below

$$
\left(I_{\text {tot }}\right)_{\max _{\min }}=I_{1}+I_{2} \pm 2 \sqrt{I_{1} I_{2}} \text {. }
$$

The relation (1) states that the maximum and minimum intensities in the fringes occur when the third term is positive and negative, respectively. These maximum and minimum are called as constructive and destructive interferences which occur according to the phase differences between two interfering beams.

A CCD camera was used to capture the photos of the fringe patterns. Figure 3 shows the fringe patterns captured using CCD camera with different concentrations. To analyse the acquired fringe patterns for four different concentrations, we have used fast Fourier transform (FFT) method and could derive the induced phase differences using MATLAB software. The nonlinear refractive index $\left(n_{2}\right)$ will be calculated using matrix of phase obtained by computer.
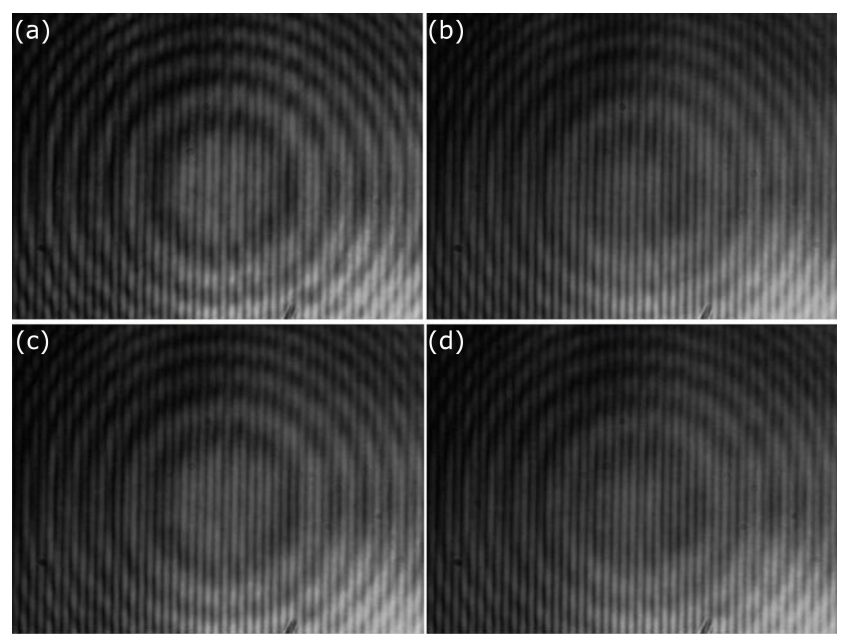

Fig. 3. Fringe patterns of aqueous $\mathrm{TiO}_{2}$ nanoparticles for: (a) 0.02 , (b) 0.04 , (c) 0.08 , (d) 0.12 molar concentrations.

\subsubsection{Z-scan technique}

As we explained earlier, the Michelson interferometer was applied to nonlinear optical investigation of aqueous $\mathrm{TiO}_{2}$ nanoparticles. This method is accurate and com- 
plete, but we preferred to use another expletive method which is reliable, to have a comparison of the obtained values of nonlinear refractive index $\left(n_{2}\right)$. So, the common Z-scan method was used as a proven technique. Actually, the Z-scan technique with close aperture is often used in studies to measure the properties of a wide variety of materials [17].

Figure 4 shows the schematics of Z-scan setup using a coherent laser beam (He-Ne, $632.8 \mathrm{~nm}$, maximum power $75 \mathrm{~mW}$ ) diverged by lens while the sample can move along one direction, so that the output power can be recorded by a power-meter. In Z-scan technique with close aperture we need an aperture before the detector and the detector is connected directly to a power meter.

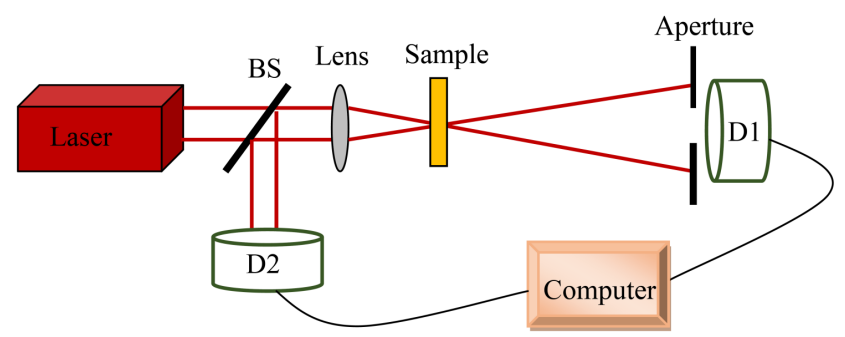

Fig. 4. The experimental setup of close Z-scan.

The nonlinear refractive index can be calculated by fitting the normalized transmittance data to the experimental results from the close aperture method that is given by [9]:

$$
T(z)=1+\frac{4 x \Delta \varphi_{0}}{\left(x^{2}+9\right)\left(x^{2}+1\right)},
$$

where $\Delta \varphi_{0}$ is the on-axis phase shift, which is defined by the following equation:

$$
\Delta \varphi_{0}=\frac{2 \pi}{\lambda} n_{2} I_{0} L_{\mathrm{eff}}, \quad L_{\mathrm{eff}}=\frac{1-\mathrm{e}^{-\alpha l}}{\alpha} .
$$

Figure 5 shows the normalized transmittance for four various concentrations of aqueous $\mathrm{TiO}_{2}$ nanoparticles. Also, the linear absorption coefficient was obtained by using the Beer-Lambert formula

$$
I=I_{0} \mathrm{e}^{-\alpha l} \text {. }
$$

In this paper, the length of sample is determined by a quartz cell of $1 \mathrm{~mm}$ length. Indeed, the effective length and peak to valley transmittance $\left(\Delta T_{p-v}\right)$ are related to the concentration of samples. Table I shows the values for linear absorption and peak to valley transmittance of the samples.

\section{Results and discussions}

The Michelson interferometer and Z-scan techniques have been applied to measure the nonlinear refractive index $\left(n_{2}\right)$ of aqueous $\mathrm{TiO}_{2}$ nanoparticles with different concentrations. In the Michelson interferometer the phase matrix of obtained fringes was calculated in MATLAB and the nonlinear refractive index was calculated and this value was compared to the Z-scan results.
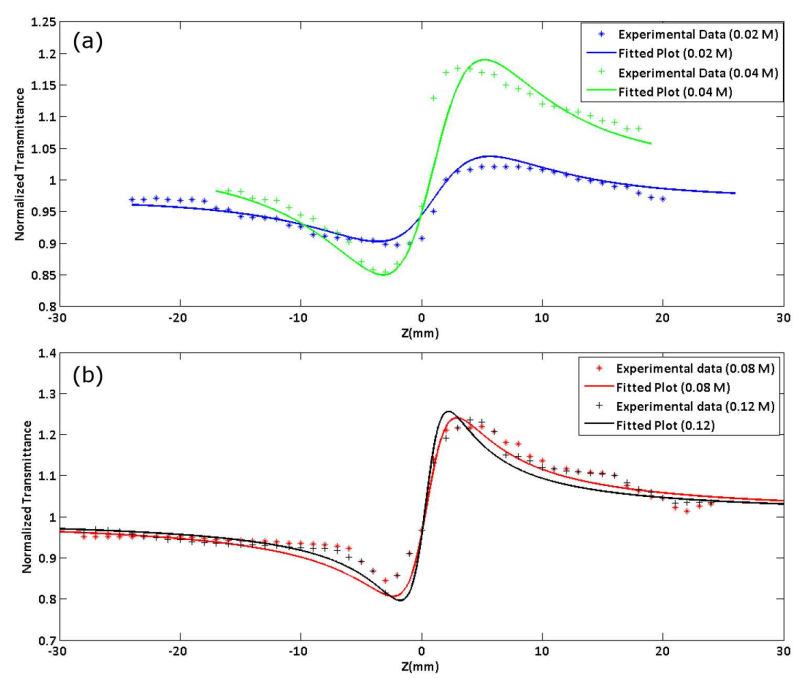

Fig. 5. The close Z-scan plots for (a) $0.02 \mathrm{M}$ and $0.04 \mathrm{M}$ and (b) $0.08 \mathrm{M}$ and $0.12 \mathrm{M}$ of aqueous $\mathrm{TiO}_{2}$ nanoparticles. The dots and lines are experimental and theoretical data, respectively.

TABLE I

The linear absorption index and peak to valley transmittance of the samples.

\begin{tabular}{c|c|c|c}
\hline \hline $\begin{array}{c}\text { Concentration } \\
\text { of } \mathrm{TiO}_{2} \\
{[\mathrm{mg} / \mathrm{ml}]}\end{array}$ & $\alpha\left[\mathrm{mm}^{-1}\right]$ & $L_{\text {eff }}[\mathrm{mm}]$ & $\Delta T_{p v}$ \\
\hline 0.02 & 0.29 & 1.86 & 0.11 \\
0.04 & 0.32 & 1.85 & 0.15 \\
0.08 & 0.65 & 1.73 & 0.36 \\
0.12 & 0.72 & 1.71 & 0.39
\end{tabular}

In this study we used a He-Ne laser source $(632.8 \mathrm{~nm})$ in both the Michelson interferometry and Z-scan setups. It should be stated that, to separate the linear refraction from the nonlinear part in interferometry method, we first captured the fringe patterns when the output power laser was reduced to less than $2 \mathrm{~mW}$ until the linear response was dominant. After that, by increasing output power to $75 \mathrm{~mW}$, the nonlinear effects appear obviously and to calculate the nonlinear refractive index, two captured patterns are diminished to eliminate the linear refraction part.

Table II shows the results for both methods. The value of nonlinear refractive index $\left(n_{2}\right)$ using interferometry method for aqueous $\mathrm{TiO}_{2}$ in $0.02,0.04,0.08$, and 0.12 molar concentrations is $0.62,1.01,1.32$ and 1.52 $\left(10^{-8}\right) \mathrm{cm}^{2} / \mathrm{W}$, respectively. Also, these values for these samples are $1.55,2.98,4.83$, and $5.97\left(10^{-8}\right) \mathrm{cm}^{2} / \mathrm{W}$ by Z-scan method. In fact, the nonlinear refractive index increases with the increase in concentration of nanoparticles for interferometry method. Also, the obtained values using Z-scan technique confirms this fact and again the $n_{2}$ increases. By plotting the change of nonlinear refractive index versus the concentration of samples for 
both methods (Fig. 6), although at first sight, it seems that the results are different in magnitude (but same in order) but the trend of graphs are the same. The other reason for the differences between $n_{2}$ values is due to the shape of incident laser beam. In Z-scan method, we focus the laser beam by a lens and therefore the spatial intensity increases, but in the interferometry method the laser beam is expanded by a lens, and therefore the spatial intensity decreases. Moreover, to explain why the nonlinear optical responses occur, it has to be noted that actually there is a temperature rise $(\Delta T)$ and additional refractive index change which is defined as: $(\Delta n)_{\text {thermal }}=\left(\frac{\mathrm{d} n}{\mathrm{~d} t}\right) \Delta T$. Since for $\mathrm{TEM}_{00}$ Gaussian laser beam, $\Delta T$ is larger around the beam axis than away from the axis, they produce a nonuniform spatial distribution of $\Delta n_{\text {thermal }}$ which leads to nonlinear optical responses.

TABLE II

The nonlinear refractive index $\left(n_{2}\right)$ of $\mathrm{TiO}_{2}$ for four different concentrations by both method.

\begin{tabular}{c|c|c}
\hline \hline $\begin{array}{c}\mathrm{TiO}_{2} \\
\text { concentration } \\
{[\mathrm{mg} / \mathrm{ml}]}\end{array}$ & $\begin{array}{c}n_{2} \text { obtained using } \\
\text { interferometry } \\
\text { method } \\
\times 10^{-8}\left[\mathrm{~cm}^{2} / \mathrm{W}\right]\end{array}$ & $\begin{array}{c}n_{2} \text { obtained using } \\
\text { Z-scan technique } \\
\times 10^{-8}\left[\mathrm{~cm}^{2} / \mathrm{W}\right]\end{array}$ \\
\hline 0.02 & 0.62 & 1.55 \\
0.04 & 1.01 & 2.98 \\
0.08 & 1.32 & 4.83 \\
0.12 & 1.52 & 5.97
\end{tabular}

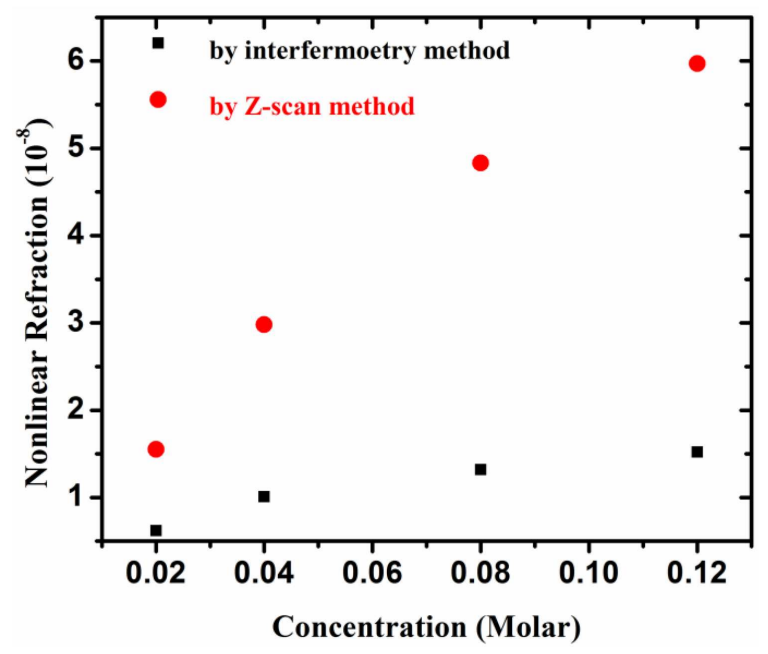

Fig. 6. The change of nonlinear refractive index versus the concentration of sample for both methods, Z-scan and interferometry method.

Finally, it seems that the interferometry method is quite applicable, because in every laboratory, a Michelson interferometry exists and by analysing the fringe patterns by MATLAB or other software, one can calculate the magnitude of nonlinear refractive index very fast. So, this method can help researcher to study the optical nonlinearity responses.

\section{Conclusion}

For the first time we have applied the Michelson interferometer for measurement of the nonlinear refractive index $n_{2}$ of aqueous $\mathrm{TiO}_{2}$ nanoparticles. The advantage of this setup over other complex methods is using a single coherent beam which leads to reducing the complexity of experimental setup. Also, to confirm the obtained results, all results were repeated again by close Z-scan method. By both methods, the value of nonlinear refractive index is of the order of $10^{-8} \mathrm{~cm}^{2} / \mathrm{W}$. In addition, the results show that the value of $n_{2}$ depends on the concentration of nanoparticles which is proved by both interferometry and Z-scan methods.

\section{References}

[1] S. Mathew, B. Samuel, A. Mujeeb, M. Kailasnath, V.P.N. Nampoori, C.P. Girijavallabhan, Opt. Mater. 72, 673 (2017)

[2] S. Jian-Hong, S. Hui-Dong, W. Zi-Wei, W. Kun, Y. Yong, L. Qi, L. Ling, Chin. Opt. 10, 760 (2017).

[3] A. Vanaja, K.S. Rao, Adv. Nanopart. 5, 83 (2016).

[4] I. Dancus, S.T. Popescu, A. Petris, Opt. Exp. 21, 31303 (2013).

[5] B.E.A. Saleh, M.C. Teich, Fundamentals of Photonics, 2nd ed., Wiley, 2007.

[6] R.R. Bommareddi, Technologies 2, 54 (2014).

[7] M.I. Abu Taha, A.S. Jabr, J. Mod. Phys. 5, 763 (2014).

[8] J.S. Santos, I.M. Raimundo, C.M.B. Cordeiro, C.R. Biazoli, C.A.J. Gouveia, P.A.S. Jorge, Sens. Actuat. 196, 99 (2014).

[9] M. Sheik-Bahae, Ali Said, Tai-Huei Wei, D.J. Hagan, E.W. Van Stryland, IEEE J. Quantum. Electron. 26, 760 (1990).

[10] Xiaobo Chen, A. Selloni, Chem. Rev. 114, 9281 (2014).

[11] Te-Wei Chiu, Yi-An Chen, Han-Chia Lee, Sheng-Zhe Hong, Ceram. Int. 41, 213 (2015).

[12] E. Johansson, M.Sc. Thesis, Chalmers University of Technology, Sweden 2004.

[13] F. Karc1, F. Karc, Dyes Pigm. 77, 451 (2008).

[14] M. Moran, CY She, R. Carman, IEEE J. Quant. Electron. 11, 259 (1975).

[15] A. Granmayeh Rad, J. Mod. Phys. 5, 280 (2014).

[16] M.H. Majles Ara, S. Salmani, S.H. Mousavi, E. Koushki, Curr. Appl. Phys. 10, 997 (2010).

[17] M.H. Majles Ara, E. Koushki, S.H. Mousavi, S. Salmani, M. Rafizadeh, A. Gharibi, Mater. Chem. Phys. 109, 320 (2008). 